\title{
Entropy optimization in flow of viscous fluid over a rotating stretchable disk
}

\author{
Muhammad Ibrahim ${ }^{1}$
}

${ }^{1} \mathrm{USTB}$

October 10, 2020

\begin{abstract}
This article investigates unsteady flow of viscous fluid flow by a rotating stretchable disk. Porous medium is considered and describe by Darcy's law. Using the flow assumption, the flow model governs by system of partial differential equation (PDE). New dimensional less variables are introduce to covet the system to dimensionless from. Finite difference method is utilized to solve dimensionless PDE's system. Effects of important flow parameters on velocity, temperature, Nusselt number and skin fraction are examined graphically. Results outcomes show that velocity decay though porosity parameter while temperature show is increasing function of Prandl and Eckert number. Skin fraction and Nusselt number increase for higher porosity parameter and Eckert number respectively.
\end{abstract}

\section{Hosted file}

Manuscript.pdf available at https://authorea.com/users/365976/articles/485902-entropyoptimization-in-flow-of-viscous-fluid-over-a-rotating-stretchable-disk 
figures/F1/F1-eps-converted-to.pdf 
figures/C1/C1-eps-converted-to.pdf 
figures/C2/C2-eps-converted-to.pdf 
figures/N1/N1-eps-converted-to.pdf 
figures/T1/T1-eps-converted-to.pdf 
figures/T2/T2-eps-converted-to.pdf 
figures/U2/U2-eps-converted-to.pdf 
figures/U1/U1-eps-converted-to.pdf 
figures/U3/U3-eps-converted-to.pdf 
figures/U4/U4-eps-converted-to.pdf 
figures/U5/U5-eps-converted-to.pdf 